$\begin{array}{r}\text { Artvin Çoruh Üniversitesi } \\ \text { Orman Fakültesi Dergisi }\end{array}$
YIII: 2019, Cilt: 20, SayI:2, Sayfa:144-149

\title{
Artemisia L. Subgen. Drancunculus (Bess.) Rydb. Taksonlarının uçucu yağ kompozisyonları ve antimikrobiyal özellikleri
}

\section{Antimicrobial properties and essential oils compositions of Artemisia L. Subgen. Drancunculus (Bess.) Rydb. Taxa}

\author{
Sevda KIRBAĞ ${ }^{1}$ iD, Eyüp BAĞCI ${ }^{1}$ iD , Şemsettin CIVELEK $^{1}$ iD , Murat KURŞAT $^{2}$ iD
}

${ }_{1}$ Fırat Üniversitesi Fen Fakültesi Biyoloji Bölümü, Elazığ Türkiye

2Bitlis Eren Üniversitesi Fen Edebiyat Fakültesi Biyoloji Bölümü, Bitlis Türkiye

\begin{tabular}{l} 
Eser Bilgisi/Article Info \\
Araştırma makalesi/Research article \\
DOI: $10.17474 /$ artvinofd.518783 \\
\hline Sorumlu yazar/Corresponding author \\
Sevda KIRBAĞ \\
e-mail: skirbag@firat.edu.tr \\
\hline Geliş tarihi / Received \\
28.01 .2019 \\
Düzeltme tarihi / Received in revised form \\
16.05.2019 \\
Kabul Tarihi / Accepted \\
17.06.2019 \\
Elektronik erişim / Online available \\
25.06 .2019 \\
Anahtar kelimeler: \\
Artemisia campestris \\
Artemisia scoparia \\
Uçucu yağ \\
Antimikrobial \\
Keywords: \\
Artemisia campestris \\
Artemisia scoparia \\
Essential oils \\
Antimicrobial
\end{tabular}

\begin{abstract}
Özet
Bu çalışmada, Türkiye'nin farklı yerlerinden toplanan Artemisia L. cinsinin Drancunculus (Bess.) Rydb. altcinsi taksonlarının uçucu yağların bileşenleri ve bunların antimikrobiyal özellikleri belirlenmiştir. Uçucu yağlar bitkilerin toprak üstü kısımlarından hidrodistilasyon metodu kullanılarak elde edilmiştir. Antimikrobiyal aktivite oyuk agar metodu kullanılarak tespit edilmiştir. Artemisia campestris L.var. campestris temel uçucu yağ bileşenleri sırasıyla naftalen (\%34.69), $\beta$-pinen (\%13.29), spatulenol (\%7.70), 2,4 hexadien-1-one (\%6.99) penta-2,4-dione (\%4.96), $\alpha$-pinen (1.65\%), limonen (\%1.51) tespit edilmiştir. Artemisia campestris var. marschalliana (Spreng.) Poljak.'da benzen (\%17.34), $\beta-$ pinen (\%10.54), spatulenol (\%10.45), 1-phenly-penta-2,4-diyn (\%8.06), karyofillen oxid (\%6.93), asenaphthylen (6.31\%), limonen (4.45\%), $\alpha$-pinen (3.70\%), $\beta$-mirsen (3.23\%) belirlenmiştir. Artemisia campestris L. var. araratica (Novopokr.) Poljak'da $\beta$-pinen (\%18.32), $\beta$-pinen (\%8.03), limonen (\%5.19), cis-osimen (\%2.87), trans- geraniol (1.93), trans-isolimonen (1.86\%), kamfor (\%1.79) olarak belirlenmiştir. Artemisia scoparia Waldst. \& Kit.'da 1-phenyl-penta-2,4-diyn (\%15.44), benzen (\%13.34), $\beta$-pinen (\%11.85), spatulenol (\%11.11), limonen (\%6.59), karyofillen oxid (\%5.99), $\alpha$-pinen (\%3.73), $\beta$-mircen (\%3.15), cis-okimen (\%2.96) tespit edilmiştir. Artemisia türlerinin uçucu yağları patojen mikroorganizmaların gelişimlerini farklı oranlarda engellemişlerdir.
\end{abstract}

\begin{abstract}
In this study, essential oil composition and their antimicrobial properties of the subgenus Drancunculus taxa belonging to the genus Artemisia collected from different regions of Turkey were determined. Essential oils were obtained from the above-ground parts of plants by using hydrodistillation method. Antimicrobial activity was determined using the agar well method. Major essential oil components of Artemisia campestris L. var. campestris were determined respectively: naphthalene (34.69\%), $\beta$-pinene (13.29\%), spathulenol (7.70\%), 2,4 hexadien -1-one (6.99\%), penta-2,4-dione (4.96\%), $\alpha$-pinene (1.65\%), limonene (1.51\%). Benzene (17.34\%), $\beta$-pinene (10.54\%), spathulenol, $(10.45 \%)$, 1-phenyl-penta-2,4-diyne (8.06\%), caryophyllene oxid $(6.93 \%)$, asenaphthylene $(6.31 \%)$, limonene (4.45\%); $\alpha$-pinene (3.70\%), $\beta$-myrsen (3.23\%) in Artemisia campestris var. marschalliana (Spreng.) Poljak. was determined. $\alpha$-pinene $(18.32 \%), \beta$-pinene $(8.03 \%)$, limonene $(5.19 \%)$, cisokimen $(2.87 \%)$, trans-geraniol (1.93), trans-isolimonene (1.86\%), camphor (1.79\%) in Artemisia campestris var. araratica Novopokr.) Poljak was found. 1-phenyl-penta-2,4-diamine (15.44\%), benzene (13.34\%), $\beta$-pinene (11.85\%), spathulenol (11.11\%), limonene (6.59\%), Caryophyllene oxid (5.99\%), $\alpha$-pinene (3.73\%), $\beta$-myrcen (3.15\%), cis-okimene (2.96\%) in Artemisia scoparia Waldst. \& Kit. was detect. The essential oils of Artemisia species prevented at different rates the development of pathogenic microorganisms.
\end{abstract}

\section{GíRiş}

Artemisia L. cinsi ülkemizde 3 altcins, 22 tür, 5 alttür ve 3 varyete olmak üzere toplam 26 taksonla temsil edilmektedir (Civelek ve ark. 2011; Güner ve ark. 2012). Pelin otu, Kâbe süpürgesi, yavşan otu, Kâbe kekiği, peygamber süpürgesi olarak bilinen, Artemisia cinsleri geleneksel Anadolu tıbbında tonik, antimalaryal, antihelmintik, antidiyabetik, yara, bronşit, ülser ve tüberküloz tedavisinde kullanıldığı bilinmektedir (Baytop, 1999). A. campestris' in çiçekleri kaynatılarak hipoglisemik, safra söktürücü, sindirim, obezite ve böcek sokmaları, iltahap önleyici, romatizma, kolesterolü düşürmek ve patojenlere karşı kullanılmıştır (Al-Snafi 2015). A. campestris' in antioxidant ve antitumor aktivitesi de tespit edilmiştir (Sefi ve ark. 2011; Akroudt ve ark. 2011). Artemisia cinsi türlerinin uçucu yağ bileşenleri ve bazı biyolojik aktiviteleri ile ilgili bazı çalışmalar yapılmıştır 
(Gilani ve ark. 2004; Kordalı ve ark. 2005; Al-Snafi 2013; Cha ve ark. 2005; Djidel ve Khennouf, 2014; Boulanouar ve ark. 2014; Belhattab ve ark. 2011; Ghorab ve ark.2013; Gucker 2007; Erel ve ark. 2012; Sing ve ark. 2009; Yao 2016).

Bu çalışmada, Türkiye de yetişen Artemisia L. cinsinin Drancunculus (Bess.) Rydb. altcinsine ait 4 taksonun esansiyel yağ kompozisyonu ve antimikrobiyal özellikleri araştırılması amaçlanmıştır.

\section{MATERYAL VE YÖNTEM}

\section{Materyal}

Çalışmada kullanılan taksonlar Türkiye'deki farklı bölgelerinden toplanmıştır (Çizelge 1). Türler, Fırat Üniversitesi, Fen Fakültesi Herbaryum'unda (FUH) muhafaza edilmektedir.

\section{Uçucu Yağların Eldesi}

Bitki örnekklerinden $100 \mathrm{gr}$ alınıp Clevenger apareyi ile su distilasyonu yöntemi kullanılarak uçucu yağlar elde edilmiştir. Uçucu yağların verimi, kompozisyonu kalitatif ve kantitatif anlamda belirlenmiştir. Uçucu yağların kimyasal analizleri, F.Ü. Fen Fak. Biyoloji Bölümü, Bitki Ürünleri ve Biyoteknolojisi Araştırma Laboratuvarında (BUBAL) bulunan GC-MS (Gaz kromatografisi-Kütle spekrometrisi) ile yapılmıştır.

\section{Gaz Kromatografisi (GC) ve Gaz Kromatografisi-Kütle Spekrofotometrisi (GC-MS) Analizleri}

Kromatografik işlemler Hewlett Packard sistemi, HPAgilent 5973 N GC- FID ve GC-MS 6890 GC sistemi kullanılarak gerçekleştirilmiştir. DB-5 MS kolon (30mx0.25 iç çaplı $0.25 \mathrm{~m}$ ) kullanılmıştır. Taşıyıcı gaz olarak helyum kullanılarak injektör sıcaklığı $250^{\circ} \mathrm{C}$., split akış hızı 1 $\mathrm{ml} / \mathrm{dk}$., GC (Gaz kromatografisi)' nin sıcaklığı $60^{\circ} \mathrm{C} 2 \mathrm{dk}$. ve $10{ }^{\circ} \mathrm{C} / \mathrm{dk}$. artışla $150{ }^{\circ} \mathrm{C}^{\prime}$ de tutulmuş ve $15 \mathrm{dk}$. aralıkla $240^{\circ} \mathrm{C}^{\prime}$ ye ulaşıldığında 5 /dk. bekletilmiştir. GC-MS' in şartları aynı şekilde uygulanmıştır. Uçucu yağlardaki bileşenlerin karakterizasyonu elektronik kütüphaneler (WILEY, NIST ve Uçucu yağ kütüphanesi) kullanılarak yapılmışır.

Çizelge 1. Artemisia L. Dracunculus (Bess.) Rydb. taksonlarının lokalite ve etiket bilgileri

\begin{tabular}{|c|c|c|c|}
\hline Takson & Toplayıcı no ve toplayıcı & Toplanma Tarihi & Toplandığı Yer \\
\hline A. campestris var. campestris & 1022 M. Kurşat & 05.09.2007 & $\begin{array}{l}\text { C2 Antalya: Korkuteli- Fethiye karayolu, Söğüt ilçesine } 7 \text { km kala, Yeşilova } \\
\text { köyü, yol kenarları, } 1430 \text { m. }\end{array}$ \\
\hline A. campestris var.marschalliana & 1073 M. Kurşat, Ş. Civelek & 23.09.2007 & $\begin{array}{l}\text { B9 Bitlis: Adilcevaz, Aydınlar köyü, Kıçgiller mezrasına } 1 \text { km kala, } \\
\text { yamaçlar, } 252 \text { m }\end{array}$ \\
\hline A. campestris var. araratica & 1013 M. Kurşat, Ş. Civelek & 01.09 .2007 & B6 Malatya: Doğanşehir, Dedeyazı köyü, Çanakcı mevkii, step, 1495 m. \\
\hline A. scoparia & 1030 M. Kurşat, Ş. Civelek & 10.09.2007 & $\begin{array}{l}\text { B4 Ankara: Polatlı karayolu, Temelli'yi } 3 \text { km geçtikten sonra, Polatlı’ya } 20 \\
\text { km kala, yol kenarları, } 796 \text { m. }\end{array}$ \\
\hline
\end{tabular}

\section{Çalışmada Kullanılan Mikroorganizmalar}

Araştırmada; Staphylococcus aureus COWAN1 Gr (+), Bacillus megaterium DSM32 $\mathrm{Gr}(+)$, Klebsiella pneumoniea FMC 5 Gr (-), Escherichia coli ATCC 25922 Gr (-), Candida albicans FMC17, C. glabrata ATCC66032, Trichophyton sp., Epidermophyton sp. kullanılmıştır.

\section{Mikroorganizma Kültürlerinin Hazırlanması ve Disk Diffuzyon Yöntemi}

Bakteriler, "Nutrient Buyyon"a aşılanarak $35 \pm 1^{\circ} \mathrm{C}$ 'de $24 \mathrm{~h}$; mayalar, "Malt Ekstrakt Buyyon'da $25 \pm 1{ }^{\circ} \mathrm{C}$ 'de $72 \mathrm{~h}$ ve dermatofitler "Glukozlu Sabouroud Buyyon" da $25 \pm 1^{\circ} \mathrm{C}^{\prime}$ de 72 saat süreyle inkübasyona bırakılmıştır. Besiyerinde gelişen kültürlerin, MacFarland (0.5) standart tüpüne göre bulanıklık ayarı yapıldıktan sonra buyyon tüplerine aktarılmıştır. Erlende steril edilen ve $45-50{ }^{\circ} \mathrm{C}^{\prime} \mathrm{ye}$ kadar soğutulan "Muller Hinton Agar", "Patato Dextrose Agar" ve "Sabouraud Dextrose Agar"a hazırlanan bakteri, maya ve dermatofitlerin buyyondaki kültürleri, \%1 oranında aşılanarak $\left(10^{6}\right.$ bakteri $/ \mathrm{ml}, 10^{4}$ maya $/ \mathrm{ml}, 10^{4}$ dermatofit/ml), iyice karıştıııldıktan sonra petrilere $15^{\prime} \mathrm{er} \mathrm{ml}$ olacak şekilde dökülerek homojen olarak dağılması sağlanmıştır. Katılaşan agarlara $6 \mathrm{~mm}$ çaplı oyuklar açılıp, bir damla besiyerinden sonra herbirine örneklerden $30 \mu \mathrm{l}$ doldurulmuştur. Plaklar, $4^{\circ} \mathrm{C}^{\prime}$ de $1.5-2$ saat bekletilip, bakteri aşılanan plaklar $37 \pm 1^{\circ} \mathrm{C}^{\prime}$ de 24 saat; maya ve dermatofit aşılanan plaklar $25 \pm 1^{\circ} \mathrm{C}^{\prime}$ de 3 gün inkübasyona bırakılmıştır. Süre sonunda inhibisyon zonları mm olarak ölçülmüştür (NCCLS, 1999). Kontrol için standart antibiyotik disklerden kullanılmıştır. 


\section{BULGULAR VE TARTIŞMA}

A.campestris var. campestris, A.campestris var. marschalliana, A.campestris var. araratica A. scoparia'nın uçucu yağ kompozisyonu Çizelge 2-5 de verilmiştir.

Çizelge 2. A. campestris var. campestris'in uçucu yağ bileşenleri

\begin{tabular}{|c|c|c|c|}
\hline No & BILEŞENLER & RT & $\%$ Oran \\
\hline 1 & $\alpha$-pinen & 7.83 & 1.65 \\
\hline 2 & Benzaldehyd & 8.81 & 0.19 \\
\hline 3 & Sabinen & 9.19 & 0.16 \\
\hline 4 & $\beta$-pinen & 9.39 & 13.29 \\
\hline 5 & $\beta$-mirsen & 9.75 & 0.21 \\
\hline 6 & Benzen & 11.01 & 0.58 \\
\hline 7 & limonen & 11.17 & 1.51 \\
\hline 8 & Cis-ocimen & 11.38 & 0.55 \\
\hline 9 & 1,3,6-octatrien & 11.75 & 0.16 \\
\hline 10 & $\gamma$-terpinen & 12.16 & 0.10 \\
\hline 11 & Acetophenon & 12.40 & 0.11 \\
\hline 12 & $\alpha$-terpinolen & 13.08 & 0.08 \\
\hline 13 & L-Linalool & 13.57 & 0.09 \\
\hline 14 & Nonanal & 13.72 & 0.08 \\
\hline 15 & $\beta$-tuyon & 13.81 & 0.13 \\
\hline 16 & Tuyon & 14.18 & 0.72 \\
\hline 17 & 3-cyclopenten-1-acetaldehyd & 14.46 & 0.21 \\
\hline 18 & Bicyclo (3.1.1) hepten-2-one & 14.86 & 0.24 \\
\hline 19 & Trans-pinocarveol & 14.94 & 0.53 \\
\hline 20 & Campfor & 15.14 & 0.46 \\
\hline 21 & 4-hexen-1-ol & 15.61 & 0.43 \\
\hline 22 & Bicyclo (3.1.0) hex-3-en-2-one & 15.82 & 0.07 \\
\hline 23 & Bicyclo (3.1.0) heptan-3-one & 16.06 & 0.03 \\
\hline 24 & 3-cyclohexen-1-ol & 16.19 & 0.18 \\
\hline 25 & Dekanal & 16.93 & 0.05 \\
\hline 26 & Trans (+) carveol & 17.35 & 0.09 \\
\hline 27 & Cis-3-hexenyl isovalerat & 17.85 & 0.06 \\
\hline 28 & 2-cyclohexen-1-one & 18.17 & 0.08 \\
\hline 29 & 2,6,10-dodecatrien & 18.35 & 0.05 \\
\hline 30 & Penta-2,4-dione & 19.69 & 4.96 \\
\hline 31 & Campfen & 22.98 & 0.12 \\
\hline 32 & 3H-3a,7-methanoazulen & 24.13 & 0.12 \\
\hline 33 & $\beta$-sesquiphellandren & 25.38 & 0.05 \\
\hline 34 & Geranylacetone & 25.58 & 0.06 \\
\hline 35 & 1H-benzocyclohepten & 25.72 & 0.17 \\
\hline 36 & 1H-cyclepro(e)azulen & 26.00 & 0.05 \\
\hline 37 & Naftalen & 27.24 & 34.69 \\
\hline 38 & Spathulenol & 29.38 & 7.70 \\
\hline 39 & Isospathulenol & 30.81 & 0.50 \\
\hline 40 & 2,4-hexadien-1-one & 31.00 & 6.99 \\
\hline 41 & $(+)$ - $\alpha$-cyperon & 33.50 & 0.44 \\
\hline 42 & Pentacosen & 37.28 & 0.63 \\
\hline 43 & Hexadekanoik acid & 38.32 & 0.13 \\
\hline \multicolumn{3}{|c|}{ Toplam } & 78.80 \\
\hline
\end{tabular}

A. campestris var. campestris major uçucu yağ bileşenleri sırasıyla naftalen (\%34.69), $\beta$ - pinen (\%13.29), spathulenol (\%7.70), 2,4-hexadien-1-one (\%6.99), penta2,4-dion (\%4.96), $\alpha$-pinen (\%1.65) ve limonen (\%1.51) tespit edilmiştir.
Çizelge 3. A.campestris var. marschalliana'nın uçucu yağ bileşenleri

\begin{tabular}{|c|c|c|c|}
\hline No & BILEŞENLER & RT & $\%$ Oran \\
\hline 1 & Bicyclo $(3,1,0)$ hex-2-ene & 7.56 & 0.06 \\
\hline 2 & $\alpha$-pinen & 7.84 & 3.70 \\
\hline 3 & Benzaldehyd & 8.82 & 0.21 \\
\hline 4 & Sabinen & 9.20 & 0.33 \\
\hline 5 & $\beta$-pinen & 9.42 & 10.54 \\
\hline 6 & $\beta$-mircen & 9.78 & 3.23 \\
\hline 7 & Benzen & 11.01 & 0.34 \\
\hline 8 & limonen & 11.19 & 4.45 \\
\hline 9 & 1,8-cineol & 11.30 & 0.97 \\
\hline 10 & Cis-ocimen & 11.39 & 0.52 \\
\hline 11 & 1,3,6-octatrien & 11.75 & 0.59 \\
\hline 12 & $\gamma$-terpinen & 12.16 & 0.14 \\
\hline 13 & $\alpha$-terpinolen & 13.08 & 0.48 \\
\hline 14 & Bicyclo $(3,1,1)$ heptan-2-one & 14.86 & 0.14 \\
\hline 15 & Trans-pinocarveol & 14.95 & 0.41 \\
\hline 16 & 4-hexen-1-ol & 15.63 & 1.29 \\
\hline 17 & 3-cyclohexen-1-ol & 16.19 & 1.03 \\
\hline 18 & Ethanon & 16.30 & 0.09 \\
\hline 19 & Trans (+)-carveol & 17.37 & 0.13 \\
\hline 20 & Cis-3-hexenyl isovalerat & 17.85 & 0.07 \\
\hline 21 & 2-cyclohexen-1-one & 18.19 & 0.09 \\
\hline 22 & 1-phenyl-penta-2,4-diyn & 19.75 & 8.06 \\
\hline 23 & Dekanoik acid & 23.06 & 0.38 \\
\hline 24 & Benzen & 24.16 & 17.34 \\
\hline 25 & Geranyl butirat & 24.58 & 0.48 \\
\hline 26 & Trans-caryophyllen & 24.74 & 1.98 \\
\hline 27 & $\beta$-selinen & 25.89 & 0.24 \\
\hline 28 & 1H-cycloprop(e)azulen & 26.01 & 0.09 \\
\hline 29 & Napftalen & 26.90 & 0.13 \\
\hline 30 & Bicyclogermacren & 27.10 & 0.79 \\
\hline 31 & Acenaphthylen & 27.22 & 6.31 \\
\hline 32 & Geranyl asetat & 27.32 & 0.54 \\
\hline 33 & $\beta$-bisabolen & 28.95 & 0.21 \\
\hline 34 & Cis-2,6-dimethyl-2,6octadien & 29.14 & 0.18 \\
\hline 35 & spathulenol & 29.47 & 10.45 \\
\hline 36 & Caryofillen oxid & 29.58 & 6.93 \\
\hline 37 & E-sesquilavandulol & 30.60 & 0.79 \\
\hline 38 & Isospathulenol & 30.82 & 0.54 \\
\hline 39 & Caryophyllenol-II & 31.77 & 0.66 \\
\hline 40 & $\alpha$-bisabolol & 32.11 & 1.43 \\
\hline 41 & e-farnesen & 33.04 & 0.16 \\
\hline 42 & 2-pentadekanon & 35.58 & 0.08 \\
\hline 43 & n-hexadekanoik acid & 38.32 & 0.03 \\
\hline 44 & Heneikosen & 42.76 & 0.03 \\
\hline 45 & Heptadekan & 47.93 & 0.03 \\
\hline \multicolumn{3}{|c|}{ Toplam } & 86.67 \\
\hline
\end{tabular}

A. campestris var. marschalliana' da benzen (\%17.34), $\beta$ pinen (\%10.54), spathulenol (\%10.45), 1-phenyl-penta2,4-diyn (\%8.06) karyofillen oxid (\%6.93), acenaphthylen (\%6.31), limonen (\%4.45), $\alpha$-pinen (\%3.70), $\beta$-mircen (\%3.23) belirlenmiştir. 
Çizelge 4. Artemisia campestris var. araratica' ya ait uçucu yağ bileşenleri

\begin{tabular}{|c|c|c|c|}
\hline No & bileşenler & RT & $\%$ Oran \\
\hline 1 & Triklen & 7.47 & 0.15 \\
\hline 2 & Bicyclo $(3,1,0)$ hex-2-ene & 7.57 & 0.23 \\
\hline 3 & $\alpha$-pinen & 7.90 & 19.32 \\
\hline 4 & Kamfen & 8.42 & 0.73 \\
\hline 5 & Sabinen & 9.21 & 0.56 \\
\hline 6 & $\beta$-pinen & 9.42 & 8.03 \\
\hline 7 & $\beta$-mirsen & 9.76 & 1.36 \\
\hline 8 & 1,3-Cyclohexadien & 1074 & 0.21 \\
\hline 9 & Benzen & 11.02 & 1.24 \\
\hline 10 & limonen & 11.19 & 5.19 \\
\hline 11 & 1,8-Sineol & 11.30 & 0.06 \\
\hline 12 & Cis-osimen & 11.40 & 2.87 \\
\hline 13 & 1,3,6-octatrien & 11.76 & 0.74 \\
\hline 14 & $\gamma$-terpinen & 12.17 & 0.72 \\
\hline 15 & $\alpha$-terpinolen & 13.08 & 0.21 \\
\hline 16 & Fencholenic aldehit & 13.24 & 0.24 \\
\hline 17 & Linalool & 13.58 & 0.16 \\
\hline 18 & 3-cyclopentene-1-acetaldehid & 14.47 & 1.05 \\
\hline 19 & Bicyclo $(3,1,1)$ heptan-2-one & 14.87 & 0.26 \\
\hline 20 & Trans-pinocarveol & 14.95 & 0.58 \\
\hline 21 & Kamfor & 15.15 & 1.79 \\
\hline 22 & p-mentha-1,5-dien-8-ol & 15.25 & 0.19 \\
\hline 23 & Bicyclo $(3,1,0)$ hex-3-en-2-one & 15.83 & 0.73 \\
\hline 24 & Bicyclo $(3,1,1)$ heptan-3-one & 16.06 & 0.04 \\
\hline 25 & 3-cyclohexen-1-ol & 16.20 & 0.54 \\
\hline 26 & Ethanon & 16.30 & 0.88 \\
\hline 27 & Benzoik asid & 16.52 & 0.07 \\
\hline 28 & Trans-isolimonen & 16.65 & 1.86 \\
\hline 29 & Dekanol & 16.93 & 0.55 \\
\hline 30 & Bicyclo $(3,1,1)$ hep-3-en-2-one & 17.02 & 0.09 \\
\hline 31 & Trans-(+)-carveol & 17.37 & 0.93 \\
\hline 32 & 6-octen-1-ol & 17.57 & 0.06 \\
\hline 33 & Cis-3 hexenyl-isovalerat & 17.85 & 0.34 \\
\hline 34 & Trans-2 hexenyl-isovalerat & 18.15 & 0.43 \\
\hline 35 & Trans-geraniol & 18.36 & 1.93 \\
\hline 36 & 1 phenyl-penta-2,4-dienyl & 19.69 & 0.23 \\
\hline 37 & Cis-2,6-dimethyl-2,6-Octadien & 22.23 & 0.42 \\
\hline 38 & Neryl-acetat & 22.56 & 0.29 \\
\hline 39 & Dekanoik acid & 23.11 & 0.75 \\
\hline 40 & Geranil acetat & 23.26 & 0.57 \\
\hline 41 & Bicyclo $(2,2,1)$ heptan & 23.52 & 0.15 \\
\hline 42 & Benzenebutanal & 24.11 & 0.07 \\
\hline 43 & $1 \mathrm{H}-3 \mathrm{a}, 7$-methano azulen & 24.20 & 0.12 \\
\hline 44 & $\beta$-caryofillen & 24.74 & 0.33 \\
\hline 45 & Germacrene D & 25.06 & 0.05 \\
\hline 46 & $1 \mathrm{H}$-cyclepro(e)azulen & 25.33 & 0.23 \\
\hline \multicolumn{2}{|c|}{ Toplam } & & 57.79 \\
\hline
\end{tabular}

A. campestris var. araratica'da $\alpha$-pinen (\%19.32), $\beta$ pinen (\%8.03), limonen (\%5.19), cis-osimen (\%2.87), trans-geraniol (\%1.93) ve trans-isolimonen (\%1.86) belirlenmiştir. A. campestris var. campestris'de toplam 43, A. campestris. var. marschalliana' da $45, A$. campestris var. araratica'da 46, $A$. scoparia 'da 43 ana bileşen tespit edilmiştir. Bileşenler ve miktarları taksonlara göre değişkenlik göstermiştir.
A. campestris'in uçucu yağında; $\beta$-pinen (\%25.6), sabinen (\%17), $\alpha$-pinen (\%9.90) (Boulanouar ve ark., 2014); $\alpha$ terpenil asetat (\%19), $\alpha$-pinen (\%18), kafur (\%9) (Belhattab ve ark., 2011); $\beta$-mirsen (\%16.47), $\alpha$-pinen (\%14.18), trans- $\beta$ - osimen (\%12.61) (Ghorab ve ark., 2013); $\beta$-pinen (\%24.2-27.9), p-simen (\%17.4-22.3) ve $\alpha$ pinen (\%4.1-11.0) (Gucker ve Corey, 2007) olarak tespit etmişlerdir.

Çizelge 5. Artemisia scoparia' ya ait uçucu yağ bileşenleri

\begin{tabular}{|c|c|c|c|}
\hline No & Bileşenler & RT & $\%$ Oran \\
\hline 1 & $\alpha-$ pinen & 7.85 & 3.73 \\
\hline 2 & Kamfen & 8.42 & 0.50 \\
\hline 3 & Sabinen & 9.22 & 0.59 \\
\hline 4 & $\beta$-pinen & 9.45 & 11.85 \\
\hline 5 & $\beta$-mirsen & 9.79 & 3.15 \\
\hline 6 & Benzen & 11.03 & 2.51 \\
\hline 7 & limonen & 11.22 & 6.59 \\
\hline 8 & 1,8 cineol & 11.31 & 0.21 \\
\hline 9 & Cis-ocimen & 11.42 & 2.96 \\
\hline 10 & 1,3,6-octatrien & 11.77 & 0.71 \\
\hline 11 & $\gamma$-terpinen & 12.18 & 0.82 \\
\hline 12 & $\alpha$-terpineole & 13.09 & 0.05 \\
\hline 13 & Cyclohexen & 14.48 & 0.20 \\
\hline 14 & Bicyclo $(3,3,1)$ heptan-2-one & 14.87 & 0.12 \\
\hline 15 & Trans-pinocarveol & 14.97 & 0.42 \\
\hline 16 & 4-hexen-1-ol & 15.65 & 0.65 \\
\hline 17 & 3-cyclohexen-1-ol & 16.21 & 0.19 \\
\hline 18 & Bicyclo $(3,3,1)$ heptan-2-ene & 16.68 & 0.74 \\
\hline 19 & 2-cyclohexen-1-ol & 17.38 & 0.12 \\
\hline 20 & 6-octen-1-ol & 17.58 & 0.05 \\
\hline 21 & 2-cyclohexen-1-one & 18.20 & 0.01 \\
\hline 22 & 1-phenyl-penta-2,4-diyn & 19.84 & 15.44 \\
\hline 23 & Bicycloelemen & 21.62 & 0.02 \\
\hline 24 & Phenol & 22.35 & 0.33 \\
\hline 25 & $(+)$ cyclosativen & 23.01 & 0.02 \\
\hline 26 & $\alpha$ copaen & 23.24 & 0.30 \\
\hline 27 & Benzen & 24.16 & 13.34 \\
\hline 28 & Trans-caryofillen & 24.77 & 2.10 \\
\hline 29 & Cis-2,6-dimethyl-2,6-octadien & 25.35 & 0.15 \\
\hline 30 & 1,6,10-dodecatrien & 25.76 & 0.21 \\
\hline 31 & $\alpha$-caryopfillen & 25.89 & 0.21 \\
\hline 32 & 1H-cycloprop(e)azulen & 26.02 & 0.06 \\
\hline 33 & $\beta$-selinen & 26.92 & 0.08 \\
\hline 34 & Bicyclogermacren & 27.10 & 0.67 \\
\hline 35 & Naphthalan & 27.22 & 2.49 \\
\hline 36 & 1,6,10-dodecatriene-3-ol & 28.96 & 0.02 \\
\hline 37 & Spathulenol & 29.50 & 11.11 \\
\hline 38 & Caryophyllene oxid & 29.60 & 5.99 \\
\hline 39 & E-sesquilavandulol & 30.60 & 0.45 \\
\hline 40 & Isospathulenol & 30.84 & 0.60 \\
\hline 41 & Caryophyllene-II & 31.77 & 0.35 \\
\hline 42 & Heneicosan & 42.77 & 0.50 \\
\hline 43 & Tricosan & 47.93 & 0.04 \\
\hline \multicolumn{2}{|c|}{ Toplam } & & 92.07 \\
\hline
\end{tabular}


A. scoparia'da; 1-phenyl-penta-2,4-diyn (\%15.44), benzen (\%13.34), $\beta$-pinen (\%11.85), spathulenol (\%11.11), limonen (\%6.59), $\alpha$-pinen (\%3.73), $\beta$-mirsen (\%3.15), cisosimen (\%2.96) ve benzen (\%2.51) belirlenmiştir.

A. scoparia ana bileşen olarak 1,2-dehydro acenaftalifitilen \%11.80 (Erel ve ark. 2012); beta-mirsen \%29.27, limonen \%3.3, Z -beta-osimen \%13.37 ve gamaterpinen \%9.51 (Singh ve ark. 2009); 2-etenil-naftalen $\% 45.1$, beta-pinen \%11.2, 3-karen \%8.7 olarak belirlenmiştir (Yao ve Bo 2016).

$\mathrm{Bu}$ çalışma sonuçları ile yukarıda ki çalışmalar karşılaştırıldığında birçok uçucu yağ bileşenlerinin (benzen, $\beta$ - pinen, spathulenol, limonen, $\alpha$-pinen, $\beta$ mirsen, cis-osimen vd.) incelenen her türün uçucu yağında bulunduğu fakat bazılarının ise türler arasında farklılık gösterdiği saptanmıştır. Uçucu yağların kimyasal ve ana bileşenlerin miktarları, coğrafi konum, mevsim, iklim değişikliği, bitki çeşitliliği ve incelenen kısımlarına göre farklılık gösterebileceği tespit edilmiştir (Akrout ve ark. 2003)

Çizelge 6. Artemisia L. cinsi taksonlarının antimikrobiyal aktiviteleri

\begin{tabular}{lccccc}
\hline \multirow{2}{*}{ Mikroorganizmalar } & \multicolumn{4}{c}{ Uçucu yağlar } & \multicolumn{2}{c}{ Standart } \\
\cline { 2 - 6 } & A.c.v.c & A.c.v.m & A.c.v.a & A.s & Tcy/Nys*/Ktc* \\
\hline E.coli & 16 & 15 & 19 & 21 & 18 \\
K. pneumoniae & 10 & 18 & 14 & 18 & 17 \\
S. aureus & 18 & 12 & 8 & 19 & 15 \\
B. megaterium & 10 & 8 & 9 & 15 & 16 \\
C. albicans & 8 & 23 & 9 & 8 & $18^{*}$ \\
C. glabrata & 8 & 18 & 17 & 15 & $12^{*}$ \\
Epidermophyton sp & 9 & 15 & 16 & 19 & 13 \\
Trichophyton spp. & 9 & 18 & - & & \\
\hline
\end{tabular}

A.c.v.c: A. campestris var. campestris, A.c.v.m: A. campestris var. marschalliana A.c.v.a: A. campestris var. araratica, A.s: A. scoparia Tcy: Tetracycline (30 $\mu \mathrm{g}$ ) Nys:Nystatin ( $30 \mu \mathrm{g} /$ disk) Ktc:Ketoconazole $(15 \mu \mathrm{g} /$ disk $)$

Bu çalışmada; uçucu yağ örnekleri kullanılan mikroorganizmaların tümünün gelişimini önlemişlerdir (8-21 mm inhibisyon zonu) (Çizelge 6). A. scoparia'nın uçucu yağlarının etkisi genellikle standarttan yüksek bulunmuştur. Ayrıca uçucu yağların dermatofitlerin (Epidermophyton sp, Trichophyton spp) gelişimlerinin engellenmesi bu çalışma ile ortaya konulmuştur. Daha önce yapılan çalışmalarda test mikroorganizmaları olarak dermatofit funguslar kullanılmamıştır. A. campestris'in uçucu yağı Gr (-) bakteri türlerine karşı aktivite göstermemesine rağmen $\mathrm{Gr}(+)$ gelişimlerini önlediği bildirilmiştir (Bnouham ve ark. 2002). Aynı türün uçucu yağlarının kullanılan patojenlerin tümünü ve en fazla $S$. aureus ile E. coli'nin gelişimini inhibe ettiği $(12 \mathrm{~mm}, 17$ mm inhibisyon zonu) belirtilmiştir (Ghorab ve ark. 2013). A. campestris ve $A$. scoparia'nın uçucu yağları kullanılan mikroorganizmaların gelişimi farklı oranlarda inhibe ettiği, en fazla Candida albicans'a karşı etkili olduğu rapor edilmiştir (20 ve $40 \mathrm{~mm}$ ihibisyon zonu) (Erel ve ark. 2012).

A. scoparia'nın uçucu yağının E. coli ve C. albicans'a karşı etkili olduğu (Coşar ve ark. 1994), S. aureus'a gelişimini engellerken $P$. aeruginosa'nın gelişimine etki etmediği belirlenmiştir (Ramezani ve ark. 2004).

Elde edilen sonuçlara göre bitki ekstraktlarının çoğu mikroorganizmalara karşı etkili antimikrobiyal maddeler ihtiva ettiği söylenebilir. Bitkilerin fitokimyasal özellikleri türden türe farklılık gösterdiği için bazı bitki ekstraktları antimikrobiyal aktiviteleri farklılık göstermekte ve bazılarında ise hiç etki göstermemektedir.

\section{TEŞEKKÜR}

Bu çalışma TÜBITAK, TBAG-106T559 tarafından desteklenmiştir.

\section{KAYNAKLAR}

Akrout AA, Chemli R, Simmonds M and Kite G (2003) Mohamed Hammami and Imed Chreif. Seasonal Variation of the Essential Oil of . L Essent. Oil Res.15:333-336

Akrout A, Gonzalez LA, El Jani H and Madrid PC (2011) Antioxidant and antitumor activities of Artemisia campestris and Thymelaea hirsuta from southern Tunisia. Food Chem Toxicol, 49(2):342-347.

Al-Snafi AE (2015) The Pharmacological Importance Of Artemisia campestris-A ReviewAsian J. Pharm. Res. 5 (2): 88-92.

Al Snafi AE (2013) Encyclopedia of the constituents and pharmacological effects of Iraqi medicinal plants. Thi qar University, 235-241

Baytop T (1999) Türkiye'de Bitkiler ile Tedavi: Geçmişte ve Bugün. Nobel Tıp Kitabevi. İstanbul.

Belhattab RR, Boudjouref M, Barroso JG, Pedro LP, Figueirido AC (2011) Essential Oil Composition from Artemisia campestris Grown in Algeria. Advances in Environmental Biology, 5(2): 429-432.

Bnouham M, Mekhfi H, Legssyer A, Ziyyat A (2002) Ethnopharmacology Forum: Medicinal plants used in the treatment of diabetes in Morocco. Int J Diabetes \& Metabolism, 10: 33-50.

Boulanouar B, Abdelaziz G, Mohamed M, and Miguel MG (2014) Chemical composition of essential oils of Artemisia campestris and Juniperus phoenicea from Algeria International Journal of Innovation and Applied Studies. 9 (4): 1434-1436.

Cha JD, Jeong MR, Jeong SI, Moon S E, Kim J Y, Kil B S, Song Y H (2005) Chemical composition and antimicrobial activity of the essential 
oils of Artemisia scoparia and A. capillaries. Planta Med. 2: 186190.

Coşar G, Çubukçu B, Melikoğlu G (1994) Antimicrobial activity of Artemisia species growing in Turkey. J Fac Pharm İstanbul 30: 1924.

Civelek Ş, Kurşat M, Bagcı E, Kırbag S, Gür N, Türkoğlu i (2011) Türkiye de Yetişen Artemisia L Asteraceae Türleri Üzerinde Taksonomik Kimyasal, Flavonoid ve Uçucu Yağlar, Karyolojik Palinolojik ve Antimikrobiyal Aktivite Araştırmaları Tübitak Proje No $106 T 559$.

Djidel S and Khennouf S (2014) Radical scavenging, reducing power, lipid peroxidation inhibition and chelating properties of extracts from Artemisia campestris L. aerial parts. Annual Research \& Review in Biology, 4(10): 1691-1702.

Erel BŞ, Reznicek G, Şenol SG, Karabay N, Yavaşoğlu Ü, Konyalıoğlu S, Zeybek AU (2012) Antimicrobial and antioxidant properties of Artemisia L. species from western Anatolia.Turk J Biol 36: 75-84.

Gilani AH, Janbaz KH, Lateef A, Zaman M (1994) Ca Channel Blocking Activity Of Artemisia scoparia Extract. Phytotherapy Research, 83 (3):161-165.

Ghorab H, Laggoune S, Kabouche A, Semra Z and Kabouche Z (2013) Essential oil composition and antibacterial activity of Artemisia campestris L. from Khenchela (Algeria). Der Pharmacia Lettre, 5(2): 189-192.

Gucker, Corey L (2007) Artemisia campestris. U.S. Department of Agriculture, Forest Service, Rocky Mountain Research Station,
Guner A, Aslan S, Ekim T, Vural M, Babac MT (2012) Türkiye bitkileri listesi (Damarlı Bitkiler). İstanbul: Nezehat Gökyiğit Botanik Bahçesi ve Flora Araştırmaları Derneği Yayınları.

Kordali S, Cakir A, Mavi A, Kilic H, Yildirim A (2005) Screening of chemical composition and antifungal and antioxidant activities of the essential oils from three Turkish Artemisia species. J. Agric. Food Chem. 53: 1408-1416.

National committee for clinical laboratory standards (NCCLS). Performance standards for antimicrobial susceptibility testing: ninth informational supplement, 1999;19:21.

Ramezani M, Fazli-Bazza BS, Saghafi -Khadem F (2004) Antimicrobial activity of four Artemisia species of Iran. Fitoterapia 75: 201-203.

Sefi M, Bouaziz H, Soudani N, Boudawara T and Zeghal N (2011) Fenthion induced-oxidative stress in the liver of adult rats and their progeny: Alleviation by Artemisia campestris. Pesticide Biochemistry and Physiology, 101(2):71-79.

Singh HP, Kaur S, Mittal S, Batish DR, Kohli RK (2009) Essential oil of Artemisia scoparia inhibits plant growth by generating reactive oxygen species and causing oxidative damage J Chem Ecol. Feb;35(2):154-62.

Yao LH and Bo HB (2016) Essential Oil Composition of Artemisia scoparia Waldst. \& Kitag from Qinghai -Tibetan Plateau of China. Journal of Analytical Sciences, Methods and Instrumentation, 6: 15. 